\begin{tabular}{|c|c|}
\hline Title & $\begin{array}{l}\text { Power-dependent spin amplification in (In, Ga) A s/GaA s quantum well via Pauli blocking by tunnel-coupled quantum } \\
\text { dot ensembles }\end{array}$ \\
\hline Author(s) & Chen, S. L.; Kiba, T.; Y ang, X. J.; T akay ama, J.; Muray ama, A . \\
\hline Citation & $\begin{array}{l}\text { A pplied physics letters, 108(15), 152103-1-152103-4 } \\
\text { https://doi.org/10.1063/1.4945740 }\end{array}$ \\
\hline Issue Date & 2016-04-11 \\
\hline Doc URL & http:/hdl.handle.net/2115/65015 \\
\hline Rights & $\begin{array}{l}\text { This article may be downloaded for personal use only. Any other use requires prior permission of the author and AIP } \\
\text { Publishing. The following article appeared in A pplied Physics Letters 108, } 152103 \text { (2016) and may be found at } \\
\text { http://dx.doi.org } 10.1063 / .4945740 \text {. }\end{array}$ \\
\hline Type & article \\
\hline File Information & 1.4945740.pdf \\
\hline
\end{tabular}

Instructions for use 


\section{AIP/Apolied Pyysises Letters}

Power-dependent spin amplification in (In, Ga)As/GaAs quantum well via Pauli blocking by tunnel-coupled quantum dot ensembles

S. L. Chen, T. Kiba, X. J. Yang, J. Takayama, and A. Murayama

Citation: Applied Physics Letters 108, 152103 (2016); doi: 10.1063/1.4945740

View online: http://dx.doi.org/10.1063/1.4945740

View Table of Contents: http://scitation.aip.org/content/aip/journal/apl/108/15?ver=pdfcov

Published by the AIP Publishing

\section{Articles you may be interested in}

Ultrafast spin tunneling and injection in coupled nanostructures of $\mathrm{InGaAs}$ quantum dots and quantum well Appl. Phys. Lett. 104, 012406 (2014); 10.1063/1.4861387

Temperature-dependent carrier tunneling for self-assembled InAs/GaAs quantum dots with a GaAsN quantum well injector

Appl. Phys. Lett. 96, 151104 (2010); 10.1063/1.3396187

Time resolved photoluminescence of $\ln (\mathrm{N})$ As quantum dots embedded in $\mathrm{Galn}(\mathrm{N}) \mathrm{As} / \mathrm{GaAs}$ quantum well Appl. Phys. Lett. 96, 041911 (2010); 10.1063/1.3299258

Electrical spin injection from $\mathrm{ZnMnSe}$ into InGaAs quantum wells and quantum dots Appl. Phys. Lett. 88, 062105 (2006); 10.1063/1.2172221

InAsN/GaAs ( N ) quantum-dot and InGaNAs/GaAs quantum-well emitters: A comparison Appl. Phys. Lett. 86, 233107 (2005); 10.1063/1.1944899

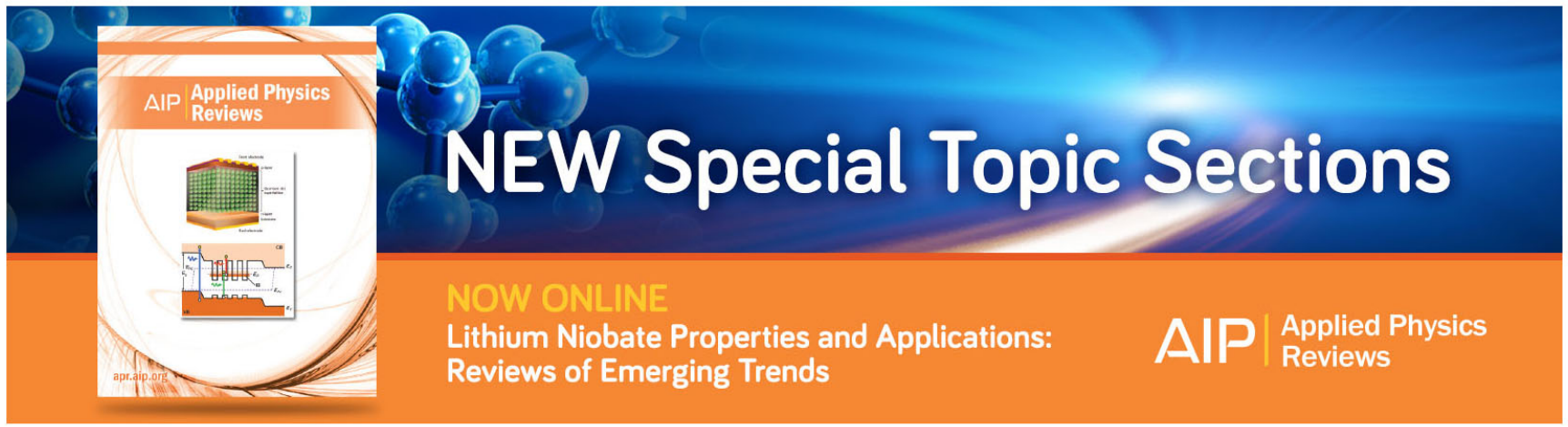




\title{
Power-dependent spin amplification in (In, Ga)As/GaAs quantum well via Pauli blocking by tunnel-coupled quantum dot ensembles
}

\author{
S. L. Chen, ${ }^{1, a)}$ T. Kiba, ${ }^{2}$ X. J. Yang, ${ }^{3}$ J. Takayama, ${ }^{1}$ and A. Murayama ${ }^{1}$ \\ ${ }^{1}$ Graduate School of Information Science and Technology, Hokkaido University, Kita 14, Nishi 9 , \\ Kita-ku, Sapporo 060-0814, Japan \\ ${ }^{2}$ Kitami Institute of Technology, 165 Koen-cho, Kitami 090-8507, Hokkaido, Japan \\ ${ }^{3}$ Suzhou QiangMing Optoelectronics Co. Ltd., Suzhou 215-028, Jiangsu, China
}

(Received 17 January 2016; accepted 29 March 2016; published online 11 April 2016)

\begin{abstract}
Power-dependent time-resolved optical spin orientation measurements were performed on $\mathrm{In}_{0.1} \mathrm{Ga}_{0.9}$ As quantum well (QW) and $\mathrm{In}_{0.5} \mathrm{Ga}_{0.5}$ As quantum dot (QD) tunnel-coupled structures with an 8-nm-thick GaAs barrier. A fast transient increase of electron spin polarization was observed at the QW ground state after circular-polarized pulse excitation. The temporal maximum of polarization increased with increasing pumping fluence owing to enhanced spin blocking in the QDs, yielding a highest amplification of $174 \%$ with respect to the initial spin polarization. Further elevation of the laser power gradually quenched the polarization dynamics, which was induced by saturated spin filling of both the QDs and the QW phase spaces. (C) 2016 AIP Publishing LLC.

[http://dx.doi.org/10.1063/1.4945740]
\end{abstract}

Quantum-coupled nanostructures have been the subject of intense research owing to the fact that composite nanosystems possess the properties of each component, which renders them an ideal platform for exploring the physics and materials functions. One example is quantum well (QW) and quantum dot (QD) tunnel-coupled nanostructures. ${ }^{1-9}$ In such hybrid systems, QWs serve as reservoirs for carriers/excitons, whereas the three-dimensional (3D) potential surrounding QDs efficiently confines the carrier/exciton motion. This confinement leads to long-lived spin states due to the suppression of spin-orbit interaction-induced relaxation processes. ${ }^{10}$ Therefore, QDs have been demonstrated to be efficient spin filters. ${ }^{11-14}$ Enhanced electron spin polarization was observed at excited states (ESs) of QDs by spin blocking from ground states (GSs). ${ }^{11,15-17}$ Although extensively studied, these previous works mainly focused on the localized spin states of the QDs. Wider application of this spin amplification effect can be found by tunnel-coupling QDs with quasi-two-dimensional (2D) mobile carriers/excitons in QWs. There are two major advantages of such hybrid structures: (i) the tunneling process is spin-conserving, and (ii) the coupling strength can be controlled by the barrier thickness and height, enabling additional quantum engineering that cannot be achieved with QWs or QDs alone. By integrating current mature QW-based opto-electronic devices with the spin-filter function of QDs, quantum-coupled nanostructures can be fabricated for use in spin-polarized light-emitting/laser diodes (LED/LDs). ${ }^{18,19}$ Therefore, by employing time-resolved optical spin orientation spectroscopy in this work, we focused on the excitation power-dependent spin amplification effect in a (In, Ga)As/GaAs QW tunnelcoupled with spin-polarized QD ensembles.

The (In, Ga)As/GaAs QW-QD coupled structure was grown by molecular beam epitaxy (MBE) on a [001]-oriented GaAs substrate with a 400-nm-thick GaAs buffer

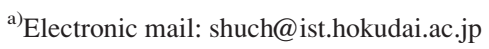

layer. Separated by an 8-nm-thick GaAs tunneling barrier, the $\mathrm{In}_{0.1} \mathrm{Ga}_{0.9}$ As QW has a thickness of $20 \mathrm{~nm}$, whereas the $\mathrm{In}_{0.5} \mathrm{Ga}_{0.5} \mathrm{As}$ QD ensembles have an average basal diameter and height of $20 \mathrm{~nm}$ and $5 \mathrm{~nm}$, respectively. An areal dot density, $n_{Q D}$, of $\sim 2 \times 10^{10} \mathrm{~cm}^{-2}$ was revealed by scanning electron microscopy (SEM). Finally, the QD layer was capped with a 50-nm-thick GaAs layer. It should be noted that the sample is slightly p-type doped due to residual carbon acceptors in the GaAs layer. Schematically shown in the inset of Figure 1(a) is the high-angle angular dark-field (HAADF) scanning transmission electron microscopy (STEM) image of the coupled structure, where the QDs and QW are outlined by dashed lines. The arrow bar denotes a scale of $20 \mathrm{~nm}$.

The time-resolved optical spin orientation study was performed at $6 \mathrm{~K}$ in a He-cycling cryostat. A mode-locked Ti:sapphire pulsed laser with a repetition rate of $76 \mathrm{MHz}$ and a temporal width of $150 \mathrm{fs}$ was used as the excitation source. To resonantly generate spin-polarized carriers inside the QW, a linear polarizer and a quarter-wave plate were employed at the excitation side, and the laser photon energy was tuned to $1.46 \mathrm{eV}$, which is below the GaAs bandgap of approximately $1.52 \mathrm{eV}$ at $6 \mathrm{~K}$. Because the QW GS wavefunction is tunnel-coupled with QDs through the thin barrier layer, the photo-excited carriers in the QW could be transferred to the dots with no loss of spin and subsequently relax to the localized QD ES. Time-resolved PL emissions from the QW and QDs were detected by a streak camera system combined with a single grating monochromator. To distinguish the circular-polarized $\left(\sigma^{+}\right.$and $\left.\sigma^{-}\right)$emission from the coupled structure, we applied another linear polarizer and quarter-wave plate on the detection side.

Figure 1 (a) shows the $6 \mathrm{~K}$ circular polarization-resolved photoluminescence (PL) spectra measured at a pumping density, $P_{\text {exc }}$, of $7.1 \mathrm{~W} / \mathrm{cm}^{2}$. The entire PL emission is strongly co-polarized with laser helicity $\left(\sigma^{+}\right)$. A relatively sharp PL transition centered at $1.413 \mathrm{eV}$ arises from the QW GS at the 


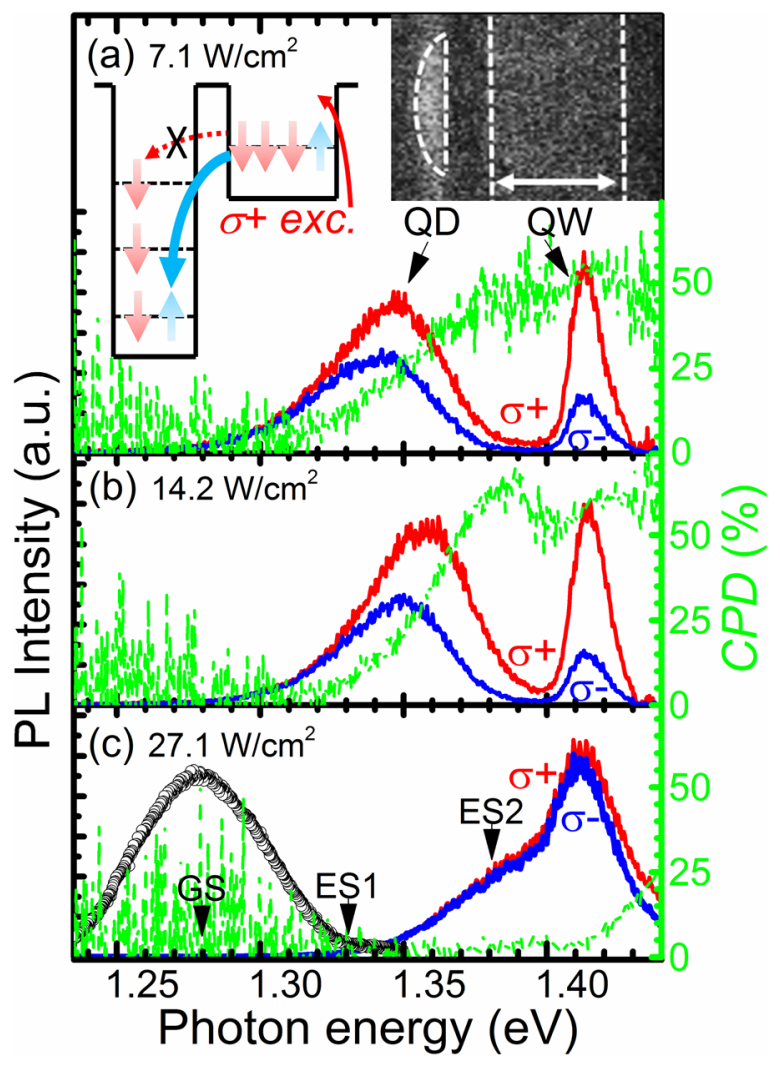

FIG. 1. $6 \mathrm{~K}$ circular-polarized PL spectra measured at the excitation density, $P_{\text {exc }}$, of (a) $7.1 \mathrm{~W} / \mathrm{cm}^{2}$, (b) $14.2 \mathrm{~W} / \mathrm{cm}^{2}$, and (c) $27.1 \mathrm{~W} / \mathrm{cm}^{2}$. The corresponding $C P D$ is given as dashed curve. Scheme for optical excitation and subsequent spin-dependent tunneling of QW GS electrons, together with HAADF-STEM image of QW-QD coupled structure, is given in (a). The arrow bar denotes $20 \mathrm{~nm}$ length scale. QD GS PL spectrum is shown as dotted line in (c).

Brillouin center $(k=0)$. Below this transition is a much broader emission from 1.30 to $1.39 \mathrm{eV}$, mainly coming from the ES emission of the QDs. Because of the response limit of the streak camera detector, the PL intensity decreases considerably toward the low energy side. By using another CCD detector from SpectrumOne with low excitation power, the GS of the QD ensembles was observed at $1.280 \mathrm{eV}$ after spectral deconvolution, as shown by the dotted line in Figure 1(c). Calculation of the QD energy levels based on Ref. 20 gave two excited states, ES1 and ES2, positioned at $1.331 \mathrm{eV}$ and $1.383 \mathrm{eV}$, respectively. Because the energy separation between QD ES2 and QW GS was only $\sim 30 \mathrm{meV}$, which is smaller than the QD inter-level spacing of $\sim 50 \mathrm{meV}$, the carriers excited in the QW were injected into the QDs mainly via the non-resonant tunneling process. By calculating the PL circular polarization degree $(C P D)$, i.e., $C P D=\left(I_{\sigma+}\right.$ $\left.-I_{\sigma-}\right) /\left(I_{\sigma+}+I_{\sigma-}\right)$, where $I_{\sigma+}$ and $I_{\sigma-}$ are the $\sigma^{+}$and $\sigma^{-}$polarized PL intensities, the QW GS gave a $C P D$ value of approximately $50 \%$. For the QD emission, its polarization decreases steadily from ES2 toward the low energy side and reaches almost zero at GS, indicating a full occupation of spin states. This feature is characteristic of the spindependent intra-level carrier relaxation, i.e., the spin blockade effect. ${ }^{11,15-17}$ Since holes in QWs experience stronger spin-orbit interaction and mixing of heavy-light hole sub-valence bands, ${ }^{10,21-23}$ their spins are easily randomized, which leaves electron spins better preserved to determine the
PL helicity of the QW and QDs. By increasing $P_{\text {exc }}$ to 14.2 W/ $/ \mathrm{cm}^{2}$ - see Figure 1(b), the $C P D$ of the QW and QD ES2 increased to $\sim 60 \%$. At the same time, the QD emission was blue-shifted, caused by enhanced state filling under higher pumping. However, further increasing the laser power drastically reduced the $C P D$ of the QDs and $\mathrm{QW}$ to almost zero at the highest $P_{\text {exc }}$ of $27.1 \mathrm{~W} / \mathrm{cm}^{2}$ - see, e.g., Figure 1(c). The QD emission then merged with the QW GS transition, resulting in the appearance of an extended "hot-carrier" PL tail over the QW GS continuum $(|k|>0)$ at the higher energy side, which is a clear sign of saturation in both the QDs and the QW. The observed power dependence of circular polarization, together with the concurrent change of the PL spectral shape, points to their close correlation.

To gain insight into the underlying spin dynamics, we turned to time-resolved PL. Shown in Figure 2(a) are the $\sigma^{+}$and $\sigma^{-}$-polarized PL transients from the QW GS at $P_{\text {exc }}$ of $7.1 \mathrm{~W} / \mathrm{cm}^{2}$. Both PL traces feature fast decay of approximately $24 \mathrm{ps}$, which was induced by rapid tunnel-injection of carriers from the QW GS to the QD ES, as reported before. ${ }^{8}$ For comparison, Figure 2(a) also includes the unpolarized PL decay (stars) from a reference QW fabricated using the same growth conditions. The reference QW shows typical radiative decay around $1 \mathrm{~ns}$. The time evolution of $C P D$ based on circular PL dynamics is displayed in Figure 2(d) as triangles. An initial $C P D, C P D_{t=0}$, of $43 \%$ is observed at the

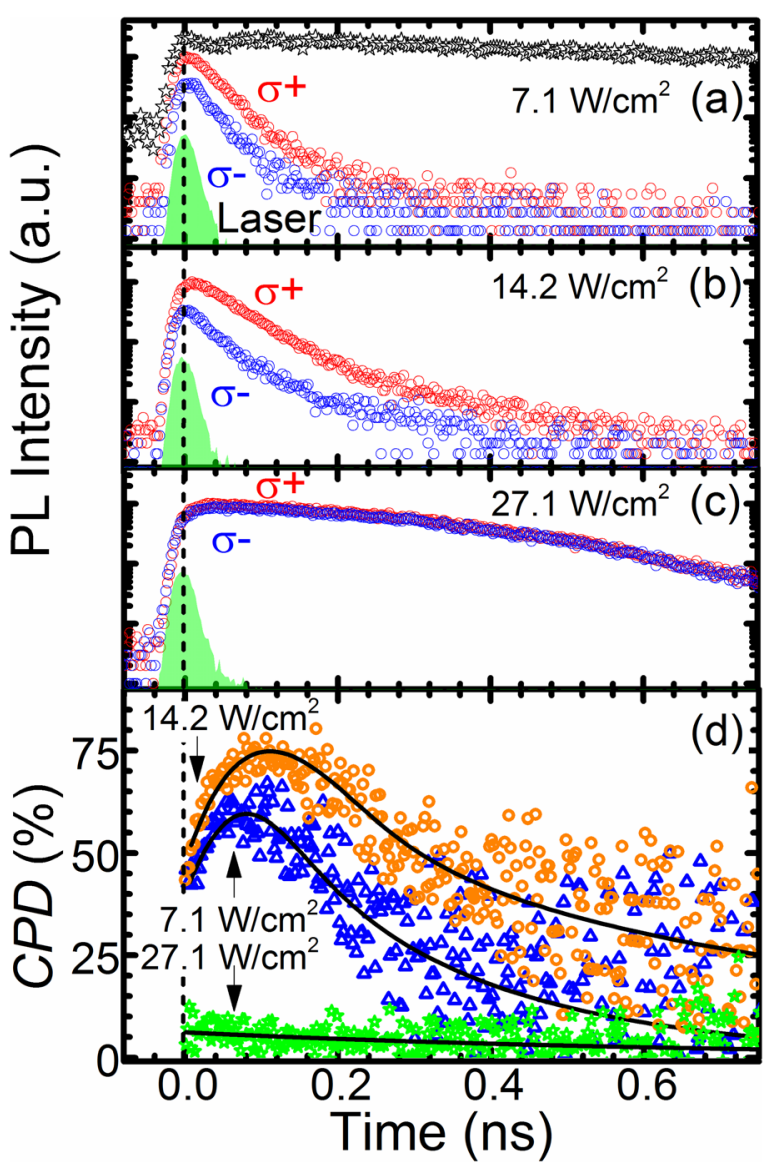

FIG. 2. (a)-(c) $6 \mathrm{~K}$ Circular-polarized time-resolved PL of QW GS as a function of $P_{\text {exc }}$. For comparison, unpolarized PL decay of a reference QW was shown as stars in (a). (d) The derived transient $C P D$ from circularpolarized PL at $P_{\text {exc }}$ of $7.1 \mathrm{~W} / \mathrm{cm}^{2}$ (triangles), $14.2 \mathrm{~W} / \mathrm{cm}^{2}$ (dots), and $27.1 \mathrm{~W} / \mathrm{cm}^{2}$ (stars), the lines are guide to the eye. 
time origin, reflecting electron spin polarization generated by laser excitation in the QW. In such a case, the $\sigma^{+}$-polarized laser at $1.46 \mathrm{eV}$ is in resonance with the heavy/light hole-electron transition with a $3: 1$ absorption intensity ratio according to the selection rule. ${ }^{10,24}$ This leads to a $50 \%$ electron spin polarization in the $\mathrm{QW}$, which is quite close to the $C P D_{t=0}$ value we observed. The discrepancy between theoretical and measured values is most likely caused by the loss of a small fraction of electron spins during energy relaxation to the QW GS. It is noteworthy that, after laser pulse, there is a fast increase of $C P D$ up to a maximum value, $C P D_{\max }$, of $62 \%$ within $\sim 100 \mathrm{ps}$, yielding an amplification factor, $C P D_{\max } / C P D_{t=0}$, of $144 \%$. The transient increase of $C P D$ can be explained by spin-dependent tunneling of electrons from the QW to the QDs, as illustrated in the inset of Figure 1(a), i.e., the majority spin-down electrons generated by the $\sigma^{+}$laser quickly occupied the corresponding spin sub-levels in the QDs after excitation, which hindered further spin injection from the QW GS via the Pauli blocking effect. However, spin-up minorities can feed into the QDs without bafflement. The combined effects of the two spin polarizations thus lead to an initial increase of $C P D$ at the QW GS. It can be inferred that a stronger spin-polarized state filling in QD ensembles would favor spin amplification at the QW GS. Indeed, at an elevated $P_{\text {exc }}$ of $14.2 \mathrm{~W} / \mathrm{cm}^{2}$-see Figure 2(b), the increased occupation of spin levels in the QDs suppressed carrier injection from the $\mathrm{QW}$, resulting in longer PL decay for both $\sigma^{+}$and $\sigma^{-}$components. Meanwhile, the relatively weaker blocking of the minority spin-up electrons led to faster $\sigma^{-} \mathrm{PL}$ decay than that for $\sigma^{+}$polarization. The resulting time-dependent $C P D$ is shown in Figure 2(d) as dots. We can see that $C P D_{\max }$ increased up to $75 \%$. Given the same $C P D_{t=0}$ of $43 \%$, a maximal transient amplification of $174 \%$ was obtained. After reaching its temporal peak, $C P D$ decayed due to electron spin relaxation at the $\mathrm{QW}$ and QD states. For the QW, spin-flip at the QW GS directly decreased $C P D$, whereas for the QDs, it weakened the Pauli blocking of the spin-down majority electrons and rebalanced the spin populations in the QW. To compare their relative contributions to the $C P D$ dynamics, we measured the spin relaxation times of QD ES/QW GS, $\tau_{Q D}^{s} / \tau_{Q W}^{s}$, within the spectral range of $1.34-1.39 \mathrm{eV} / 1.41-1.42 \mathrm{eV}$ from coupled structure/reference single QW. The results are displayed in Figure 3(a) as squares and stars for QD ES and QW GS, respectively. The value of $\tau_{O D}^{s}, \sim 0.32 \mathrm{~ns}$, is found much faster than $\tau_{Q W}^{s}, \sim 0.8 \mathrm{~ns}$, implying its dominant role in $C P D$ decay at the QW GS. Actually, the PL circular polarization decreased monotonically with a characteristic time of $\sim 0.30 \mathrm{~ns}$, which is in good agreement with $\tau_{Q D}^{s}$. It should be noted that when both spin sub-spaces in the QDs become occupied, the $C P D$ decay of the QW GS will become longer, and it will be mainly governed by $\tau_{Q W}^{s}$. The transient increase of $C P D$ is closely dependent upon spin polarization in the QDs. This amplification effect diminishes as both the spin-down and spin-up states in the QW GS and QDs are gradually saturated-see, e.g., Figure 2(c). At $P_{e x c}$ of $27.1 \mathrm{~W} / \mathrm{cm}^{2}$, the $\sigma^{+}$and $\sigma^{-}$PL transients from the QW GS $(k=0)$ overlay each other and exhibit a saturated decaying profile, which is evidence of phase space filling and is consistent with the "hot-carrier" tail of the QW GS shown in

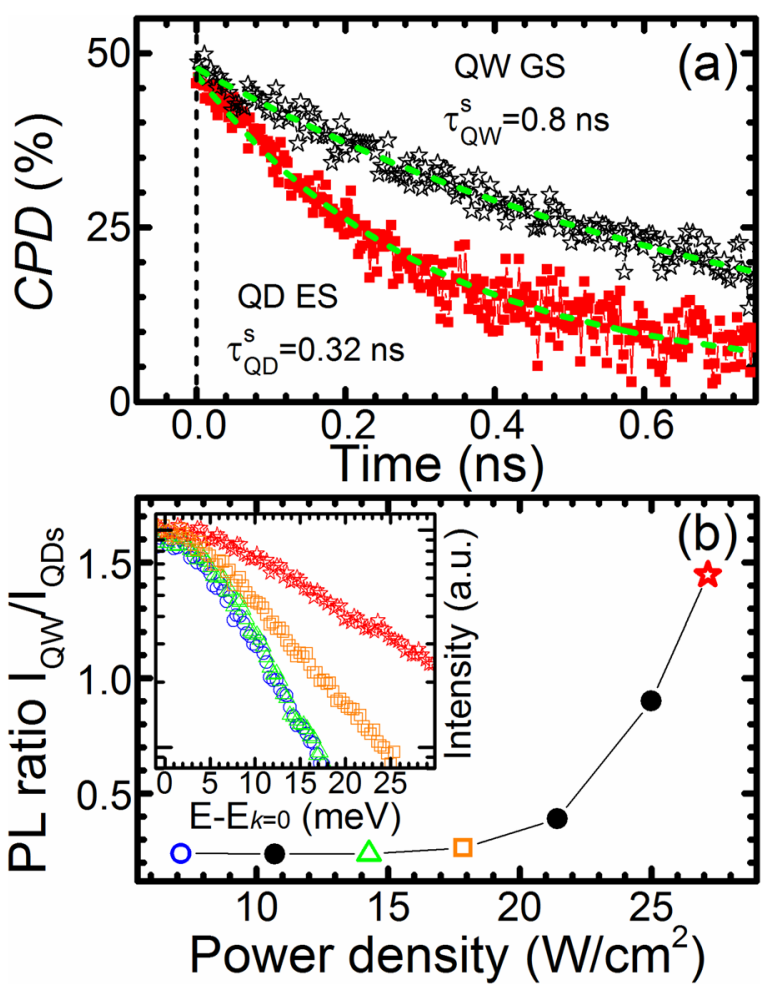

FIG. 3. (a) $C P D$ decay measured from the GS of reference QW (stars) and QD ES (squares), which reveals electron spin relaxation time of $0.8 \mathrm{~ns}\left(\tau_{Q W}^{s}\right)$ and $0.32 \mathrm{~ns}\left(\tau_{O D}^{s}\right)$. The dashed lines are single-exponential fittings to the $C P D$ decay. (b) PL intensity ratio of $\mathrm{QW}$ GS to ensemble $\mathrm{QDs}, \mathrm{I}_{\mathrm{QW}} / \mathrm{I}_{\mathrm{QD}}$, versus $P_{\text {exc }}$. Inset shows the normalized PL tail spectra of QW GS at corresponding $P_{e x c}$ with same symbols. All spectra are displayed in relative energy to their PL peaks.

Figure 1(c). As a result, $C P D_{t=0}$ is markedly reduced to $\sim 6 \%$, shown as stars in Figure 2(d), without an increase in polarization, i.e., $C P D_{t=0}=C P D_{\max }$.

Detailed power-dependent measurements were performed to obtain the relationship between optical pumping and polarization amplification. Shown in Figure 3(b) is the QW/QD PL ratio as a function of $P_{\text {exc }}$. A threshold is found at approximately $15 \mathrm{~W} / \mathrm{cm}^{2}$, which separates the power profile into unsaturated and saturated regimes. This transition of carrier occupation is corroborated by two factors. The first factor is the spectral change of the PL tail at the QW GS, as logarithmically plotted in the inset of Figure 3(b) for unpolarized detection. All spectra are normalized and displayed at energies relative to their peak at $k=0$. The corresponding excitation power is indicated by the same symbol in Figure 3(b). As can be seen, the PL tail at $7.1 \mathrm{~W} / \mathrm{cm}^{2}$ (dots) and $14.2 \mathrm{~W} / \mathrm{cm}^{2}$ (triangles) remains almost the same. It begins to increase when $P_{e x c}$ is increased to $17.8 \mathrm{~W} / \mathrm{cm}^{2}$ (squares) and finally gets populated with "hot" carriers at $27.1 \mathrm{~W} / \mathrm{cm}^{2}$ (stars). The second factor is the consistent $P_{\text {exc }}$ dependence of $C P D_{t=0}$, as shown in Figure 4(a), i.e., the initial polarization decreases as $P_{e x c}$ exceeds $15 \mathrm{~W} / \mathrm{cm}^{2}$. Having identified the state of carrier filling in the coupled structure, we now summarize its effect on the amplification of spin polarization. In the unsaturated regime, $P_{\text {exc }}<15 \mathrm{~W} / \mathrm{cm}^{2}$, stronger optical pumping intensifies the spin blocking of the QDs, giving rise to a larger polarization value, $C P D_{Q D}$, as shown in Figure 4(b). Driven by the enhanced blockade, both 


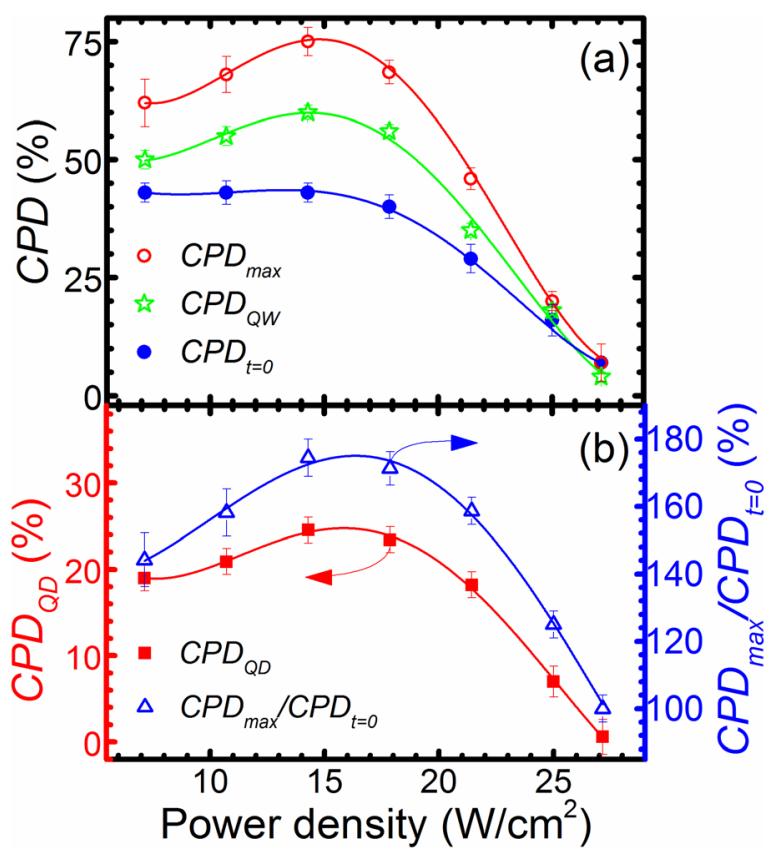

FIG. 4. (a) $P_{e x c}$ dependence of initial, $C P D_{t=0}$, temporal maximal, $C P D_{\max }$, and time-integrated, $C P D_{Q W}$, circular polarization of QW GS in coupled structure. (b) $P_{e x c}$ dependence of time-integrated circular polarization, $C P D_{Q D}$, of entire $\mathrm{QD}$ emission, together with the polarization amplification factor $C P D_{\max } / C P D_{t=0}$.

transient $C P D_{\max }$ and time-integrated polarization, $C P D_{Q W}$, of the QW GS increase with increasing $P_{\text {exc }}$, reaching a peak at approximately $P_{e x c}=14.2 \mathrm{~W} / \mathrm{cm}^{2}$. Beyond the $P_{e x c}$ threshold of $15 \mathrm{~W} / \mathrm{cm}^{2}$, the $C P D$ value of the QW GS decreases monotonically as spin saturation becomes increasingly important in the coupled structure. The amplification factor of the transient circular polarization, $C P D_{\max } / C P D_{t=0}$, is plotted against $P_{e x c}$ in Figure 4(b). It follows quite well the power dependence of $C P D_{Q D}$, manifesting the pivotal role of spin-dependent blockade in boosting the spin polarization in the QW.

In conclusion, time-resolved optical spin orientation spectroscopy was employed to study the spin amplification effect in (In, Ga)As/GaAs QW-QD tunnel-coupled nanostructures. Transient amplification of $C P D$ at the QW GS was observed via Pauli blocking by spin-polarized QD ensembles, which can be tuned by laser power. The results presented in this work demonstrate the possibility of such zerodimensional (0D)-2D hybrid quantum structures as optically controllable amplifiers for spin-based device applications.
The authors acknowledge the financial support from the Japan Society for the promotion of Science (JSPS) by Grantin-Aid for Scientific Research (S) No. 22221007, as well as the bilateral program promoted by JSPS.

${ }^{1}$ Y. I. Mazur, V. G. Dorogan, E. Marega, Jr., D. Guzun, M. E. Ware, Z. Y. Zhuchenko, G. G. Tarasov, C. Lienau, and G. J. Salamo, J. Appl. Phys. 113, 034309 (2013).

${ }^{2}$ C. Y. Jin, S. Ohta, M. Hopkinson, O. Kojima, T. Kita, and O. Wada, Appl. Phys. Lett. 96, 151104 (2010).

${ }^{3}$ Y. I. Mazur, V. G. Dorogan, E. Marega, Jr., Z. Y. Zhuchenko, M. E. Ware, M. Benamara, G. G. Tarasov, P. Vasa, C. Lienau, and G. J. Salamo, J. Appl. Phys. 108, 074316 (2010).

${ }^{4}$ D. Guzun, Y. I. Mazur, V. G. Dorogan, M. E. Ware, E. Marega, Jr., G. G. Tarasov, C. Lienau, and G. J. Salamo, J. Appl. Phys. 113, 154304 (2013).

${ }^{5}$ M. Syperek, J. Andrzejewski, W. Rudno-Rudzinski, G. Sek, J. Misiewicz, E. M. Pavelescu, C. Gilfert, and J. P. Reithmaier, Phys. Rev. B 85, 125311 (2012).

${ }^{6}$ Y. I. Mazur, V. G. Dorogan, D. Guzun, E. Marega, Jr., G. J. Salamo, G. G. Tarasov, A. O. Govorov, P. Vasa, and C. Lienau, Phys. Rev. B 82, 155413 (2010).

${ }^{7}$ M. Syperek, P. Leszczynski, J. Misiewicz, E. M. Pavelescu, C. Gilfert, and J. P. Reithmaier, Appl. Phys. Lett. 96, 011901 (2010).

${ }^{8}$ X. J. Yang, T. Kiba, T. Yamamura, J. Takayama, A. Subagyo, K. Sueoka, and A. Murayama, Appl. Phys. Lett. 104, 012406 (2014).

${ }^{9}$ D. Dagnelund, I. A. Buyanova, W. M. Chen, A. Murayama, T. Furuta, K. Hyomi, I. Souma, and Y. Oka, Phys. Rev. B 77, 035437 (2008).

${ }^{10}$ M. I. Dyakonov, Spin Physics in Semiconductors (Springer, Berlin, Heidelberg, 2008).

${ }^{11}$ M. W. Taylor, E. Harbord, P. Spencer, E. Clarke, G. Slavcheva, and R. Murray, Appl. Phys. Lett. 97, 171907 (2010).

${ }^{12}$ P. Recher, E. V. Sukhorukov, and D. Loss, Phys. Rev. Lett. 85, 1962 (2000).

${ }^{13}$ R. Hanson, L. M. K. Vandersypen, L. H. Willems van Beveren, J. M. Elzerman, I. T. Vink, and L. P. Kouwenhoven, Phys. Rev. B 70, 241304(R) (2004).

${ }^{14}$ J. A. Folk, R. M. Potok, C. M. Marcus, and V. Umansky, Science 299, 679 (2003).

${ }^{15}$ V. K. Kalevich, M. Paillard, K. V. Kavokin, X. Marie, A. R. Kovsh, T. Amand, A. E. Zhukov, Y. G. Musikhin, V. M. Ustinov, E. Vanelle, and B. P. Zakharchenya, Phys. Rev. B 64, 045309 (2001).

${ }^{16}$ A. Tackeuchi, R. Ohtsubo, K. Yamaguchi, M. Murayama, T. Kitamura, T. Kuroda, and T. Takagahara, Appl. Phys. Lett. 84, 3576 (2004).

${ }^{17}$ T. Kiba, X. J. Yang, T. Yamamura, Y. Kuno, A. Subagyo, K. Sueoka, and A. Murayama, Appl. Phys. Lett. 103, 082405 (2013).

${ }^{18}$ M. Holub and P. Bhattacharya, J. Phys. D: Appl. Phys. 40, R179 (2007).

${ }^{19}$ D. D. Awschalom, D. Loss, and N. Samarth, Semiconductor Spintronics and Quantum Computation (Springer, 2002).

${ }^{20}$ A. Wojs, P. Hawrylak, S. Fafard, and L. Jacak, Phys. Rev. B 54, 5604 (1996).

${ }^{21}$ T. Uenoyama and L. J. Sham, Phys. Rev. Lett. 64, 3070 (1990).

${ }^{22}$ T. Uenoyama and L. J. Sham, Phys. Rev. B 42, 7114 (1990).

${ }^{23}$ R. Ferreira and G. Bastard, Phys. Rev. B 43, 9687 (1991).

${ }^{24}$ F. Meier and B. P. Zakharchenya, Optical Orientation (North-Holland, Amsterdam, 1984). 